\title{
Das letzte Gefecht um den »Kriegsverrat« im NS-Staat
}

63 Jahre nach dem Ende des nationalsozialistischen Terrorregimes debattiert der Deutsche Bundestag noch immer darüber, ob eine Gruppe der Opfer der Wehrmachtjustiz einer Rehabilitierung würdig ist: die sog. Kriegsverräter. Der Versuch, diejenigen, die dem NS-Regime die Loyalität verweigerten, auch den verbrecherischen Angriffskrieg nicht mehr mitmachen wollten, zu diffamieren, hat eine lange Vorgeschichte. Nach dem Krieg zog der ehemalige Major ErnstOtto Remer unter dem Beifall Ewiggestriger durch bundesdeutsche Städte und beschimpfte die hingerichteten Verschwörer des 20. Juli 1944 als »Landesverräter «, die der deutschen Wehrmacht heimtückisch in den Rücken gefallen seien. Auf eine von dem Braunschweiger Generalstaatsanwalt Fritz Bauer erhobene Anklage wurde diesem Treiben durch das Urteil des Landgerichts Braunschweig vom I 5 . März I 952 zwar ein juristisches Ende gesetzt. Damit war der Unrechtscharakter der nationalsozialistischen Justiz aber längst nicht außer Streit. Vor allem in der Politik und im Bewusstsein der Bundeswehr ist die Anerkennung des Widerstandes gegen das NS-Regime bis heute nicht fest verankert.

Die Soldaten, die sich an dem Massenmord nicht mehr beteiligen wollten und deshalb zum Tode verurteilt worden sind, mussten mehr als ein halbes Jahrhundert auf ihre Rehabilitierung warten. Erst in der Ära der rot-grünen Koalition gelang es mit dem Unrechtsbeseitigungsänderungsgesetz von 2002, die Urteile der Wehrmachtjustiz nicht nur gegen die »Wehrkraftzersetzer « aufzuheben, sondern auch die Deserteure zu rehabilitieren. Pauschal aufgehoben wurden auch die wegen Landesverrat $(\mathbb{S}$ I I StGB) ergangenen Urteile. Von der Öffentlichkeit kaum bemerkt, blieb eine Gruppe aber ausdrücklich ausgenommen: die »Kriegsverräter«, also diejenigen, die wegen »Landesverrat im Kriege« ( $\$ 57$ Militärstrafgesetzbuch) verurteilt worden waren. Dies, obgleich ein verbrecherischer Angriffs- und Vernichtungskrieg wie der Zweite Weltkrieg von vorn herein nicht verratsfähig ist.

Ein Ende 2006 im Deutschen Bundestag eingebrachtes Gesetz möchte das Versäumte nachholen. Selten ist eine Gesetzesinitiative so fundiert begründet worden. Der Antrag stützt sich auf die von Wolfram Wette und Detlef Vogel herausgegebene Veröffentlichung »Das letzte Tabu. Kriegsverrat «. ${ }^{\mathrm{I}}$ Wolfram Wette hat darin alle in aufwändiger Recherchierungsarbeit noch zu findenden Todesurteile gegen »Kriegsverräter « veröffentlicht und analysiert, mit dem erstaunlichen Ergebnis: Was uns in den einschlägigen Kriegsgerichtsurteilen entgegentritt, ist eine Fülle unterschiedlicher unbotmäßiger, widerständiger und humaner Handlungen von Soldaten der Wehrmacht, aber kein einziger Fall wirklichen Verrats. Die Verurteilten waren Opfer einer überhart und selbst nach den nationalsozialistischen Gesetzen rechtsbeugend urteilenden Willkürjustiz.

Dennoch wollen die Fraktionen der CDU und FDP eine Rehabilitierung dieser Opfer der Wehrmachtjustiz auf keinen Fall zulassen. Nach ihrem Willen müssen jeder Verurteilte oder seine Hinterbliebenen sich einer Prüfung speziell des Einzelfalles durch die Staatsanwaltschaft stellen. Sonst, so der CSU-Abgeordnete Norbert Geis, würde man »simple verbrecherische Verräter« mit wirklichen Widerstandskämpfern auf eine Stufe stellen.

I Wolfram Wette/Detlef Vogel (Hrsg.), Das letzte Tabu. Kriegsverrat, Berlin (Aufbauverlag) 2007, zu beziehen auch über die Bundeszentrale für politische Bildung. 
Für das Hearing vor dem Rechtsausschuss des Bundestages am 5. Mai 2008 hatten CDU und FDP zwar keinen ehemaligen Kriegsrichter auftreiben können, im Unterschied zu der Anhörung, als es im Jahre I998 um die Rehabilitierung der Wehrkraftzersetzer ging. Damals hatte die CDU als »unabhängigen« Sachverständigen mit Otfried Keller einen hochrangigen und erheblich belasteten Wehrmachtjuristen präsentiert. Aber auch im Jahre 2007 konnte man mit zwei Wissenschaftlern von ähnlicher Verdrängungsmentalität aufwarten: Professor Rolf-Dieter Müller, Wissenschaftlicher Direktor des Militärgeschichtlichen Forschungsamtes, und Professor Sönke Neitzel von der Universität Mainz. Um dem Antrag der Linksfraktion auf eine pauschale Aufhebung der Urteile entgegentreten zu können, benötigten die Rehabilitierungsgegner wenigstens ein einziges Urteil, das sich auch nach heutigen Maßstäben aufrechterhalten lässt. Das von Professor Müller gelieferte Paradebeispiel, das die Abgeordneten beeindruckte und auch vom SPIEGEL (Nr. I 8 v. 28.4.2008) unbesehen übernommen wurde: ein Urteil des Reichskriegsgerichts gegen General Feuchtinger. Der habe einen Kriegsverrat höchst verbrecherischer Art begangen. Er habe seiner Freundin, einer Tänzerin, in einem Brief mitgeteilt, mit welchen Aufgaben er bei der Ardennenoffensive befasst war, und er habe ihr Pelzmäntel und eine Benzinbezugsmöglichkeit verschafft. Eine Aufhebung des Urteils gegen Feuchtinger, so Müller, wäre ein Skandal.²

Der von Müller geschilderte Sachverhalt machte mich stutzig. Die Handlungen erfüllten nicht den Tatbestand des Kriegsverrats, also des Verrats eines militärischen Geheimnisses an einen Feind oder der Sabotage. Eine Geliebte ist weder ein Feind noch eine ausländische Macht, wie dies $\ 9 \mathrm{Ib}$ StGB und $\ 57$ Reichsmilitärstrafgesetzbuch erforderten. Nach diesem Sachverhalt konnte das Reichskriegsgericht Feuchtinger allenfalls wegen Wehrkraftzersetzung verurteilt haben.

Einem in Rechtsfragen ahnungslosen Historiker könnte man einen juristischen Irrtum nachsehen, den man einem Jurastudenten im zweiten Semester wohl nicht durchgehen lassen würde. Doch es kam für Müller noch peinlicher: Die Verurteilung des Generals Feuchtinger ist allein der Phantasie des Sachverständigen entsprungen.

Wie in einem Kriminalfall kam die Wahrheit erst nach und nach ans Licht: Von mir um eine Kopie des Urteils oder wenigstens um die Angabe eines Aktenzeichens oder einer Archiv-Signatur gebeten, verwies Müller mich wortkarg an das Militärarchiv in Freiburg. Rechnete er vielleicht damit, dass der so abgespeiste Anfrager resignieren würde? Ich ließ im Militärarchiv recherchieren und erhielt die Auskunft, ein solches Urteil sei dort nicht bekannt. Also wiederholte ich meine Bitte an Müller, nun etwas nachdrücklicher. Aus der von ihm jetzt endlich genannten Quelle, nämlich einer Stellungnahme eines ehemaligen Richters am Reichskriegsgericht, Dr. Block, ergibt sich, dass Feuchtinger wegen Wehrkraftzersetzung, also nicht wegen Kriegsverrat, verurteilt worden ist. Das hätte Müller übrigens schon dem nicht nur Militärhistorikern wohlbekannten Buch von Otto Peter Schweling »Die deutsche Militärjustiz in der Zeit des Nationalsozialismus« (herausgegeben von Erich Schwinge) entnehmen können. Übrigens ist Feuchtinger bereits aufgrund des Unrechtsbeseitigungsänderungsgesetzes von 1998 rehabilitiert worden, im Unterschied zu den vielen Soldaten, die das

2 Wegen der Einzelheiten zu dem ungewöhnlichen Vorfall im Rechtsausschuss und der Intervention des Autors vgl. Ossietzky, Zweiwochenschrift für Politik, Wirtschaft, Kultur Nr. 23 vom i 5.I I.2008 sowie DER SPIEGEL Nr. 5 vom 26.I.2009 und http://www.kramerwf.de/Kriegsverrat.209.0.html. 
Reichskriegsgericht in absoluter Willkürrechtsprechung als Kriegsverräter verurteilt hat. In all diesen Fällen diente der Kriegsverratsparagraph als Terrorinstrument der Wehrmachtjustiz zur Ausschaltung von Kriegsgegnern und sonst politisch Missliebigen.

Der Vorwurf, Wolfram Wette habe in seinem Buch eine selektive Fallauswahl getroffen, fällt also voll auf den Sachverständigen Müller zurück. Mit weiteren Urteilen wegen Kriegsverrat konnten die Experten Müller und Neitzel nicht aufwarten. Stattdessen behaupten sie ins Blaue hinein, das Vorhandensein noch nicht entdeckter Urteile sei »nicht auszuschließen. "Verurteilungswürdig gewesen wegen besonders üblen Kriegsverrats - auf Kriegsverrat stand allein die Todesstrafe - seien insbesondere die »zum Feind übergelaufenen « Soldaten. Vermutlich hätten sie nach dem Frontwechsel über die Lage der deutschen Frontstellungen berichtet und damit »in einer verbrecherischen Weise den eigenen Kameraden geschadet «, ja sie »in einer Vielzahl von Fällen in Lebensgefahr gebracht«, eine »nach allen Maßstäben der zivilisierten Welt in höchstem Maß verwerfliche« Handlungsweise (so der rechtspolitische Sprecher der CDU/ CSU-Bundestagsfraktion Norbert Geis). Behauptungen dieser Art sind weder durch ein kriegsgerichtliches Urteil noch sonst belegt. Allerdings erinnern sie fatal an die in den ersten Nachkriegsjahren von rechtsradikaler und konservativer Seite ausgestreute Diffamierung der Deserteure und »Verräter«. Der Reichskriegsgerichtsrat Manfred Roeder fabulierte anlässlich seines Verfahrens bei der Staatsanwaltschaft Lüneburg3 von durch Verrat umgekommenen 200.000 deutschen Soldaten. All diese Behauptungen sind schon deshalb irrelevant, weil es für die Frage einer pauschalen Aufhebung der Todesurteile wegen Kriegsverrat einzig und allein darauf ankommt, ob es Verurteilungen wegen eines nicht ehrenhaften Kriegsverrats gegeben hat. Auch für Fälle des sog. Überlaufens haben die Rehabilitierungsgegner keine einzige solche Verurteilung nachweisen können. In den von Wolfram Wette ausgewerteten Verfahren, die gegen 39 Personen wegen »Kriegsverrats « geführt wurden, konnten keine Anhaltspunkte für eine Lebensgefährdung von anderen Soldaten gefunden werden. Ob sich der »Kriegsverrat « in der Rettung von Jüdinnen und Juden oder Kriegsgefangenen oder in Unmutsäußerungen über den ihnen sinnlos erscheinenden Krieg oder durch das »Überlaufen zum Feind « ausdrückte, in allen ermittelten Fällen sind die Taten dem politischen oder ethischen Widerstand zuzuordnen.

Trotz ihrer juristischen Irrelevanz lohnt sich eine nähere Befassung mit den Argumenten der Rehabilitierungsgegner. Sie bedeuten einen Rückfall in von der seriösen Geschichtswissenschaft überwundene Versuche zur teilweisen Ehrenrettung der Wehrmacht und der Wehrmachtjustiz. Es scheint so, als solle die quälend lange Geschichte der Rehabilitierung der Wehrmachtdeserteure fortgesetzt werden. Noch 1996 und I 998 wurde ihnen eine vorbehaltlose Anerkennung versagt. An der Spitze der Rehabilitierungsgegner stand schon damals Norbert Geis, ein unverdrossener Vertreter der Legende von einer weitgehend integer gebliebenen Wehrmachtjustiz. Weil viele Fahnenflüchtige sich »in höchst verwerflicher und krimineller Art von der Truppe entfernt« hätten, würde man mit einem »Persilschein für Deserteure (...)»denjenigen Richtern in der Militärjustiz Unrecht tun, die mit großem Mut dem Druck von Partei und Gestapo widerstanden« hätten. Man werde sich »nicht dafür hergeben, all denen neues

3 Vgl. dazu Helmut Kramer, "Landesverrat hat immer und zu allen Zeiten als das schimpflichste Verbrechen gegolten«. Das Verfahren der Staatsanwaltschaft Lüneburg von I95 I gegen den Generalrichter a.D. Manfred Roeder, in: Wette/Vogel (Fn. I), S. 69 ff. 
Unrecht zuzufügen, die in den vergangenen so Jahren von Stasi, Wolff und Co. in die Nazi-Ecke gestellt worden sind «. Das gelte »insbesondere für Hans Filbinger «. 4 Mit dieser Aufwertung der Täter und Abwertung der Opfer bewegt sich Norbert Geis in den Denkkategorien der NS-Zeit und auf einer Linie mit dem schrecklichen Urteil des Bundesgerichtshofs vom 24. Juni i 964.5 Damals hatte der Bundesgerichtshof den Angehörigen eines zum Tode verurteilten Kriegsdienstverweigerers Wiedergutmachungsansprüche mit der Begründung aberkannt, bei der Beurteilung der Rechtslage müsse »davon abgesehen werden, dass es der nationalsozialistische Staat war, der den Angriffskrieg führte«. Der Sachverhalt müsse vielmehr so betrachtet werden, »als handele es sich dabei um Vorgänge, die sich in einem Rechtsstaat abgespielt haben«. Das Recht auf Kriegsdienstverweigerung könne »nicht so weit gehen, Handlungen zu rechtfertigen, die eine ernste Gefahr für jeden Staat bedeuten«. Der Vorwurf, die »Überläufer« hätten ihre Kameraden gefährdet, gerät in eine bedenkliche Nähe zu einer Art neuer Dolchstoßlegende. Und er steht im Widerspruch zu der Rehabilitierung aller Deserteure durch das Unrechtsbeseitigungsänderungsgesetz von 2002. Mit dieser Kriminalisierung des Frontwechsels in dem nationalsozialistischem Vernichtungskrieg wird die Möglichkeit, sich der weiteren Teilnahme am Massenmord zu entziehen, auf die Flucht ins Hinterland der Front eingeengt, dies verbunden mit der absehbaren Ergreifung durch die »Kettenhunde « und anschließender kriegsgerichtlicher Verurteilung. Dank seiner späten Geburt brauchte der Bundestagsabgeordnete Norbert Geis freilich seine moralische Selbstgewissheit niemals unter den Bedingungen des mörderischen und selbstmörderischen NS-Krieges auf die Probe zu stellen. Als einer, der keinen Wehrdienst geleistet hat, musste er allerdings auf das große Erlebnis echter soldatischer Kameradschaft verzichten.

Vor allem haben die Rehabilitierungsgegner den verbrecherischen Charakter des deutschen Vernichtungskrieges völlig aus dem Auge verloren. Jeder deutsche Soldat, der sich dem Weiterkämpfen entzog, leistete einen Beitrag zur Verkürzung des Krieges und zur Beendigung des ohne die deutsche Wehrmacht nicht möglichen millionenfachen Massenmordes. Die Reduzierung der Problematik auf die hypothetische Annahme einer Lebensgefährdung deutscher Soldaten durch die Überläufer verkennt die Realität. Allein in der deutschen Wehrmacht starben im Jahr I939/40 rund I02.000, im Jahre I94 I 357.000, im Jahr I 942 572.000 , im Jahr I 943 8I 2.000, im Jahr I 944 I.802.000 und von Januar bis Mai I 945 I. 540.000 Soldaten. ${ }^{6}$ In den Jahren 1942 bis 1945 »fielen « also täglich über 3.800 deutsche Soldaten. Dazu kamen (nach unterschiedlichen Schätzungen) die 400.000 bis 600.000 Bombenkriegsopfer auf deutscher Seite. Was soll da das vage Spekulieren mit der Möglichkeit einer Kameradengefährdung?

Und darf nicht danach gefragt werden, was jeder Tag der Kriegsverlängerung für die übrige Welt bedeutete, für die Millionen dem Massenmord ausgelieferter Juden, für die Häftlinge in Konzentrationslagern? Bei insgesamt 55 Millionen Kriegstoten starben allein in der Sowjetunion nach (belegten) neuen Forschungen etwa 27 Millionen Menschen, die meisten davon als Opfer deutscher Verbrechen. Das von dem von den Sachverständigen Müller und Neitzel unterstützte Verdikt über die »Überläufer« läuft in der Konsequenz auf die Forderung hin-

4 Vgl. Pressedienst CDU/CSU-Fraktion im Deutschen Bundestag vom 2. Juni I 995.

5 Deutsche Richterzeitung 1964, S. 3 I 2 ff.

6 Vgl. Rüdiger Overmans, Deutsche militärische Verluste im Zweiten Weltkrieg, München (Oldenbourg Verlag) I 999. 
aus, jeder deutsche Soldat hätte durch ein Verbleiben in der Wehrmacht den mörderischen und selbstmörderischen Krieg weiterhin fördern müssen.

\section{Zur missbräuchlichen Verwendung des Begriffs der Kameradschaft}

Bemerkenswert ist auch die Verabsolutierung des Begriffs der Kameradschaft. Damit wollen die Rehabilitierungsgegner für die Wehrmachtsoldaten eine über die unter allen Menschen selbstverständliche Rücksichtnahme hinausgehende Pflichtenlage begründen. Hier verharren die Rehabilitierungsgegner, mit Unterstützung der von ihnen bestellten Sachverständigen, in einer ebenso technokratisch verkürzten wie undemokratischen und vorkonstitutionellen militärischen Denkwelt, weit entfernt von den Wertvorstellungen des Grundgesetzes. Kann es wirkliche, eine besondere ethische Pflichtenlage begründende Kameradschaft in einem Zwangsverband geben? Mit Mitgliedern, die zur Teilnahme an einem mit Massenmord verbundenen Angriffskrieg gezwungen werden? Die unter der Androhung, selbst vor das Kriegsgericht gestellt zu werden, ihre Kameraden wegen defätistischer Äußerungen anzeigen und in Erschießungspeletons Kameraden erschießen müssen? Wie es um die Kameradschaft in der Wehrmacht wirklich bestellt war, lässt sich auch an aus freien Stücken erfolgten Denunziationen ablesen, auch an Fällen von Lynchjustiz gegen Antifaschisten in der Gefangenschaft. Da wurden sogar ohne Zusammentreten eines Kriegsgerichts missliebige Kameraden aufgrund eines Mehrheitsbeschlusses erschossen oder sonst »umgelegt «, also ermordet.7 Auch im Verhältnis zwischen den höheren Offizieren und den Wehrmachtjuristen, die unter angenehmeren Lebensbedingungen meist ihre Haut retten konnten, zu den einfachen Soldaten kann von kameradschaftlichen Bindungen kaum die Rede sein. Hier werden Begriffe wie Kameradschaft, vaterländische Pflichterfüllung und andere Sekundärtugenden zu hohlen Suggestivformeln und zum bloßen Disziplinierungsinstrument missbraucht.

\section{Widerstand im Unrechtsstaat: Privileg für die Funktionseliten?}

Hinter der auf unbelegte Verdächtigungen gestützten Verdammung der »Kriegsverräter « verbirgt sich eine tiefgehende Geringschätzung widerständigen Verhaltens einfacher Bürger überhaupt. Es handelt sich um die Wiederaufnahme der in der Adenauerzeit geführten gespenstischen Debatte um die Zulässigkeit von Widerstand gegen den Nationalsozialismus. Als ehrenhaft und zulässig galt damals wie heute den Rehabilitierungsgegnern nur der von den gesellschaftlichen Eliten geleistete Widerstand. Anerkannt wurde zunächst nur der militärische Widerstand des 20. Juli. Schon in den Wiedergutmachungsdebatten des Jahres 1952 ging es im Kern darum, ob jede Widerstandshandlung moralisch anerkannt und materiell entschädigt werden sollte oder nur solche, die in einem, im konservativen Sinne verstandenen »nationalen Interesse « waren. In einäugiger Wahrnehmung oppositioneller Handlungen und mit einer selektiven Wiedergutmachungspraxis wurde der Widerstand der »kleinen Leute ausgegrenzt. Schon die nationalsozialistische Justiz, insbesondere auch die Wehrmachtjustiz,

\footnotetext{
7 Vgl. DER SPIEGEL Nr. 47 vom I7.I I.I949 (zum Fall Alfred Schoetz) und Manfred Messerschmidt, Die Wehrmachtjustiz 1933 bis I945, Paderborn 2005, S. 422 f. (zu Vorgängen in einem Kriegsgefangenenlager in Australien).
} 
hatte gegenüber dem aus Arbeiterkreisen geleisteten Widerstand eine besonders scharfe Verurteilungs- und Strafzumessungspraxis gehandhabt.

Demgegenüber zählte die mutige Tat des »kleinen Mannes«, so die des Georg Elser am 8. November 1939 im Münchener Bürgerbräukeller nichts, wurde sogar geschmäht. Viele Wiedergutmachungsansprüche einfacher Bürger, die sich etwa durch kritische Äußerungen oppositionell betätigt hatten, wurden abgewiesen, selbst wenn »wehrkraftzersetzende« Äußerungen mit der Todesstrafe geahndet worden waren. Eine Änderung trat insoweit erst mit der Entscheidung des Bundessozialgerichts vom I I. September I99I ein. Im Sinne dieser Sortierung des Widerstandes nahm, mit Ausnahme rechtsradikaler Kreise, niemand an dem Verrat der deutschen Angriffspläne durch höhere Offiziere (u.a. Oberstleutnant Hans Oster, Generaloberst Ludwig Beck) Anstoß. Wenn es nach dem Willen der Rehabilitierungsgegner geht, soll aber der Makel des Unwerturteils die »Überläufer « und die anderen »Kriegsverräter« weiterhin belasten. Ihnen oder ihren Hinterbliebenen wird zugemutet, sich dem unwürdigen Verfahren einer Gewissensprüfung im Einzelfall zu stellen.

Parallelen zu der Mentalität der Nachkriegsjahre gibt es viele. In seinem auch sonst an Widersprüchen reichen Gutachten meint Professor Rolf-Dieter Müller, mit den von ihm unterstellten Verratshandlungen hätten die zum Gegner überlaufenden deutschen Soldaten »erhebliche Verluste unter der eigenen Truppe verursacht, damit aber »dem militärischen Apparat allenfalls nur taktische und kurzzeitige Nachteile« zugefügt. Damit übernimmt Müller die elitäre Arroganz, mit der der einflussreiche Präsident des Bundesgerichtshofs und ehemaliges Mitglied des nationalsozialistischen Reichsgerichts Hermann Weinkauff und der unter seinem Einfluss stehende Bundesgerichtshof das Recht zum Widerstand mit einer Fülle von Anforderungen unter anderem davon abhängig machten, dass der Betreffende sich (aufgrund seiner herausgehobenen Position?) »ein klares und sicheres Urteil « haben und sich zutrauen dürfe, »dass der Widerstand Erfolg haben und die Sache zum Besseren wenden werde«. Deshalb wollten sie »Einzeldesertionen « keinesfalls anerkennen, ungeachtet des verbrecherischen Charakters des nationalsozialistischen Krieges. Diskutabel, so der Bundesgerichtshof in dem sog. Huppenkothen-Urteil vom 19.6.1956, sei ohnehin nur der »aus ernst zu nehmenden Kreisen stammende « Widerstand. Ganz in diesem Sinne möchten Rolf-Dieter Müller und Norbert Geis noch heute dem »Schützen Arsch « das Widerstandsrecht, ja sogar das Recht zur bloßen Verweigerung absprechen. Nach ihrem vordemokratischen Militärverständnis hat der einfache Soldat nur zu funktionieren, ohne jedes Nachdenken über den Sinn selbst eines verbrecherischen Angriffskriegs.

Gegenüber einer solchen selektiven Wahrnehmung des Widerstandes ${ }^{8}$ gehören zum nationalsozialistischen Widerstand auch oppositionelle Verhaltensweisen des »einfachen Volkes «, also auch die kleineren Verweigerungsformen im Alltagsleben. Mit Recht ist der hessische Generalstaatsanwalt Fritz Bauer dem BGH-Präsidenten Herrmann Weinkauff und dem Bundesgerichtshof unter Zurückweisung der konservativen Widerstandsprivilegien entgegengetreten. Denn jeder, der im Rahmen seiner Möglichkeiten dem NS-Regime oppositionell entgegentrat, zum Beispiel als Soldat einen sei es noch so geringen Beitrag zum Zusammenbruch der deutschen Front und damit zur Beendigung des nationalsozialistischen Massenmordaktionen leistete, beging eine gute Tat.

8 Wegen weiterer Einzelheiten zu diesem »Zweierlei Maß «vgl. Helmut Kramer, Das letzte Gefecht um den »Kriegsverrat« im Dritten Reich, in: Blätter für deutsche und internationale Politik, Heft 3/2009. 
Welches Menschenbild und Staatsverständnis verbirgt sich hinter der Geringschätzung des Widerstandes der »kleinen Leute«? Es ist das tradierte bürgerliche Gesellschaftsmodell, in dem für die unteren Schichten kein Platz für politische Mitwirkung ist. Indem man die Herrschaft in der Gesellschaft und sogar das Recht zur Opposition selbst gegen ein Unrechtsregime allein den Eliten zugesteht, spricht man dem widerständigen Bürger die Eignung ab, uns ein Vorbild zu sein. Was Not tut, ist aber das Bewusstsein, dass Kritik an und die Gegenwehr gegen politische und gesellschaftliche Fehlentwicklungen die Sache aller Bürger in solidarischem Handeln ist. Widerspruch gefordert ist nicht zuletzt gegen die trotz aller Erfahrungen des vorigen Jahrhunderts ungebrochene Tendenz machthungriger Politiker, Kriege vorzubereiten, zu führen oder daran mitzuwirken.

\section{Zum Diskussionsstand im Bundestag}

Während die Fraktionen der CDU/CSU und der FDP ziemlich festgelegt erscheinen, tun sich die SPD-Politiker mit dem Rehabilitierungsanliegen schwer. In der Sache gibt es inzwischen kaum noch einen SPD-Abgeordneten, der die pauschale Rehabilitierung der Kriegsverräter ablehnt. Doch hat die SPD ein doppeltes Problem. Die Schwierigkeiten liegen zum einen in der ohnehin immer wieder strapazierten Koalitionsdisziplin. Auf der anderen Seite ist der Antrag von der Linksfraktion eingebracht worden, der man auch nicht den kleinsten Finger reichen möchte. Insgeheim bedauert man bei der SPD, dass man den Antrag nicht selbst eingebracht hat. Die Bundesjustizministerin Brigitte Zypries hatte schon bei der Eröffnung der Ausstellung zur Wehrmachtjustiz am 2 I. Juni 2007 unter Berufung auf die Untersuchung von Wolfram Wette einen Anlass gesehen, »neu darüber zu diskutieren, ob man nicht auch die Verurteilung wegen Kriegsverrats pauschal aufheben sollte«. Noch deutlicher hielt sie bei der Übergabe der Ausstellung »Justiz und Nationalsozialismus « an das Land Berlin im Oberverwaltungsgericht Berlin-Brandenburg am I6. Juni 2008 es für »konsequent, auch Kriegsverrat in die lange Liste der Delikte aufzunehmen, bei denen NS-Urteile nicht mehr im Einzelfall auf ihren Unrechtscharakter geprüft werden müssen, sondern pauschal aufgehoben sind. Wer den Widerstand gegen das NS-Regime für legitim hält und wer den verbrecherischen Charakter der nationalsozialistischen Strafjustiz erkennt, der kann am Unrechtsgehalt dieser Urteile nicht zweifeln«.

Mit dem selbst verordneten Berührungsverbot bewirkt die SPD das Gegenteil dessen, was sie erreichen möchte, nämlich sich ihre Entscheidungen nicht von der Linken diktieren zu lassen: Indem sie sogar solche Vorschläge der Linken zurückweist, hinter denen ihre Abgeordneten in der Sache voll stehen, macht sie sich, vor der Öffentlichkeit sichtbar, erst recht von der Linken abhängig. Und der Vorwurf des einzig und allein von parteitaktischen Motiven veranlassten Handelns trifft dann beide Seiten, soweit er überhaupt immer passt.

Mit dem Hinweis, die Forderung nach einer Bereinigung von NS-Unrecht sei von der falschen Seite aus unredlichen Motiven gekommen, haben Politik und Justiz der Bundesrepublik schon einmal eine schwere Hypothek der Jahre 1933 bis I 945 fortbestehen lassen: In den fünfziger und sechziger Jahren deckte die DDR-Führung in propagandistischer Absicht die mangelnde strafrechtliche Verfolgung von NS-Tätern und deren Weiteramtieren in hohen Positionen der Bundesrepublik auf, insbesondere die personellen Kontinuitäten von »furchtbaren Juristen«, darunter übrigens besonders viele aus den Reihen der Wehrmachtjustiz. Anstatt das Problem in aller Gelassenheit nach den Geboten des Rechtsstaats zu lösen, wollte 
man sich vom Osten »nichts vorschreiben lassen « und ging weiterhin nur unwillig an die Aufarbeitung. Schon im Jahre I95 I hatte die SPD-Abgeordnete Maria Meyer-Sevenich im niedersächsischen Landtag erklärt: »Entnazifizierung ist nichts anderes als ein Mittel zur Bolschewisierung des westdeutschen Raumes«. Aus Hunderten von Verfahrenseinstellungen der fünfziger und sechziger Jahre gegen NS-Juristen ein einziges Beispiel: Der Landgerichtsdirektor Dr. Kurt Bellmann hatte in Prag I Io Todesurteile gefällt. In der Einstellungsbegründung von I96 I wies die Staatsanwaltschaft Hannover die von der VVN und tschechischen Verfolgtenvereinigungen gestellten Anzeigen mit folgender Begründung zurück: »Es handelt sich offenbar um eine Schützenhilfe der VVN, gelenkt vom östlichen Weltkommunismus, in ihrem Kampf gegen die westlichen Demokratien. (...) Die Anzeigen gehen also auf die gleichen Quellen zurück und sind nichts anderes als Kampfmittel im Kampfe des östlichen Weltkommunismus gegen die westlichen Demokratien und müssen als solche gesehen, erkannt und gewertet werden «.

Heute, mehr als ein halbes Jahrhundert danach, droht erneut die Gefahr, dass die SPD sich zum Gefangenen eines verhängnisvollen Reflexes macht, nämlich des ungeschriebenen Grundsatzes »jeder links von uns kommende Vorschlag ist abzulehnen, so richtig er in der Sache auch sein mag."

Zwischendurch schien sich für die SPD und damit für die Koalition eine Möglichkeit zu eröffnen, sich mit einem juristischen Kunstgriff aus der Affäre zu ziehen. Waren nicht, wenn auch unfreiwillig, von der Rehabilitierung der einfachen Landesverräter ( $\ 9 \mathrm{I} b \mathrm{StGB}$ ) auch die Kriegsverräter erfasst? Dann könnte man mit einem einfachen Beschluss des Bundestages den Antrag der Linksfraktion für gegenstandslos erklären. Tatsächlich ist dieser Notausgang versperrt. Der Kriegsverratsparagraph ( $\mathbb{S} 57$ Militärstrafgesetzbuch) fehlt in der abschließend zu verstehenden Aufzählung von NS-Unrechtsgesetzen in den Katalogen der Unrechtsbeseitigungsänderungsgesetze von 1998 und 2002. Das ergibt sich nicht nur aus einer Stellungnahme des Wissenschaftlichen Dienstes des Bundestages, sondern wird auch durch die federführenden Parlamentarier der Koalitionäre bestätigt. Nach den in der Bundstagssitzung vom ıo. Mai 2007 für ihre Fraktionen abgegebenen Erklärungen von Norbert Geis und Carl-Christian Dressel hat der Gesetzgeber damals bewusst davon abgesehen, Verurteilungen wegen Kriegsverrat per se als nationalsozialistisches Unrecht zu qualifizieren und die Urteile wegen Kriegsverrat pauschal aufzuheben, stattdessen habe er es bewusst bei der Einzelfallprüfung belassen.

Vielleicht gibt es in dieser parteitaktisch festgefahrenen Sache aber doch eine Lösungsmöglichkeit. Es geht um eine besonders ernsthafte Sache vor dem Hintergrund der nationalsozialistischen Vergangenheit. Zwar gehört der Konflikt zwischen Überzeugungstreue und Parteidisziplin mit dem Instrument des Fraktionszwanges längst zum Alltag der modernen Parlamente. Gerade bei der SPD wird diesmal aber nicht eine Fraktionsminderheit durch eine Fraktionsmehrheit überstimmt. Vielmehr steht die SPD-Fraktion inhaltlich voll hinter dem Rehabilitierungsantrag. Bei der CDU/CSU und FDP halten zwar die Wortführer in dieser Sache vehement an der Ablehnung fest. Ob aber alle ihre Fraktionskollegen ihr archaisches Denken teilen, ist nicht ausgemacht. Wer möchte sich denn schon gleichsam zu einer Art Hehler der unseriösen Gutachten der Sachverständigen Müller und Neitzel machen, insbesondere der skandalösen Vorspiegelung eines Kriegsverratsurteils gegen den General Feuchtinger?

Der Deutsche Bundestag sollte dem unwürdigen Zögern endlich ein Ende setzen und mit der pauschalen Rehabilitierung der »Kriegsverräter « eine überfällige geschichtliche Hypothek abtragen. 